\title{
Numerical prediction of the printable density range of lattice structures for additive manufacturing
}

\author{
Niyazi Tanlak ${ }^{\mathrm{a}, \mathrm{b}, *}$, Dirk Frederik De Lange ${ }^{\mathrm{a}, \mathrm{c}}$, Wim Van Paepegem ${ }^{\mathrm{a}}$ \\ ${ }^{a}$ Mechanics of Materials and Structures, Department of Materials Science and Engineering, Ghent University \\ Technologiepark-Zwijnaarde 903, 9052 Zwijnaarde, Belgium \\ ${ }^{b}$ SIM M3 Program, Technologiepark 935, B-9052 Zwijnaarde, Belgium \\ ${ }^{c}$ Universidad Autónoma de San Luis Potosi, Facultad de Ingeniería, Zona Universitaria, San Luis Potosí, Mexico
}

\begin{abstract}
Structured cellular structures are nowadays printed using additive manufacturing methods like powder bed fusion. The relative density of the cellular structures has a big role in the suitability of a lattice for printing due to the minimum printable radius constraint and powder being trapped inside an inclusion. In this work, the theoretical limits of the printable range of relative density of different lattice types are found based on the cell size using computer methods by leaving other process parameters for further research as the current parameters are the most basic ones. The results are approximated using simple polynomials to enable practical usage.
\end{abstract}

Keywords: Additive manufacturing, 3D printing, powder bed fusion, structured cellular structures, lattice, printable relative density

\section{Introduction}

Cellular structures (a.k.a. foams) are known to have a good strength to weight ratio. They are used in many engineering applications ranging from passive safety measures to space applications. By introducing structured foams, engineers have better control in tailoring the overall material properties according to their needs. However, structured cellular materials are hard to produce with conventional manufacturing methods. Nonetheless, there are studies about the fabrication of

\footnotetext{
${ }^{*}$ Corresponding author. Tel.: +32 93310446 .

E-mail address: niyazi.tanlak@ugent.be
} 
periodic cellular materials with conventional methods, such as sheet forming, perforated/slotted sheet folding/drawing [1-4], extrusion [5], wire assembly [6, 7], and investment casting methods [812], however, these techniques have limited flexibility with respect to structure type and dimensions. On the other hand, additive manufacturing (AM) allows an improved flexibility in geometry and size of lattice types. That opens up new possibilities for engineering applications.

Based on engineers' needs, the unit cell of a structured foam is tailored such that the global properties of the edifice are adjusted to fulfill the specifications. Type, size and volume fraction of the unit cell (lattice) are subjected to adjustments to reach the desired properties. Although there are studies about the mechanical properties of foams $[8,9,12-38]$, the printable ranges of the volume fraction of different lattice types with various cell sizes are not known thoroughly. Intuitively, it is tempting to think that such a cellular structure is always printable. However, the printable density limits may change dramatically with respect to the cell size and the type of lattice. Either the lower limit or the higher limit of printable density may be paramount according to the needs at hand. The lower printable density limit is based on the minimal feature size that can be printed by a specific process. The maximum printable density limit is principally based on the density up to which all raw material can be evacuated after manufacture. Although the entrapment of raw material formally allows the manufacture, the entrapped material will add mass in comparison with the designed density, which is considered unacceptable. For better customization, the limits of the feasibility of printing should be known beforehand. In this work, we will focus on selective powder bed fusion or sintering, but can equally be applied to several other AM processes.

The printability of lattice structures using powder bed fusion depends on unit cell shape, relative density (i.e. strut radius) of unit cell, strut inclination, powder particle size, powder material, machine precision, laser power, laser spot size, laser scanning speed, layer thickness, and hatch spacing. The effect of spacing between parallel laser scans (a.k.a. hatch spacing) on manufacturability was studied by Zhang et al [39]. They concluded that hatch spacing must be greater than the laser spot size. They also advised that three times bigger pore diameter than the maximum powder size must be present because partially melted powder particles stick on strut surfaces.

Sing et al. [40] reported that laser scan speed and laser power did not affect Young's modulus. 
But strut diameter or lattice type affected the elastic constant significantly while they did not affect the dimensional accuracy. They also reported that with the increase of laser scan speed or laser power, the powder adhesion on the struts decreased for Ti6Al4V. Loh et al. [41] found similar results. Other researchers like Yan et al. [42], Van Bael et al. [43], Pattanayak et al. [44] also reported strut diameter increase due to powder adhesion. Qiu et al. [45] studied the effect of laser scanning speed and laser power on strut size, strut morphology, and surface structures. They compared two different laser power $(150$ and $400 \mathrm{~W})$. $400 \mathrm{~W}$ resulted in thicker powder adhesion to struts with bigger deviation with respect to the laser power of $150 \mathrm{~W}$ for AlSi10Mg. Besides, they also found out that the scanning speeds below $3000 \mathrm{~mm} / \mathrm{s}$ affected the strut diameter. Tsopanos et al. [46] reached similar results. They showed that high laser power led to thicker struts of lattices.

Van Bael et al. [43] claimed three reasons for the deviations from the CAD model: inadequately chosen beam offset, staircase effect, and loose powder adhesion to struts. Wang et al. [47] marked the powder adhesion to struts as unavoidable in selective laser melting (SLM). The overhanging struts increase the problem. Optimizing design and process control can minimize powder adhesion. Powder adhesion is linked to balling phenomenon giving rise to beads on laser melted surfaces on building direction front by Yan et al. [42]. Abele et al. [48] claimed that building orientation has not any meaningful effect on the printability of scaffolds in SLM processes. They also claimed that powder adhesions have not any meaningful effect. On the other hand, Sallica-Leva et al. [49] concluded that powder adhesion can act as stress concentrators, which in turn affects fatigue strength of the porous structures. Hazlehurst et al. [50] concluded that surface waviness variation and heterogeneities have a big effect on the stiffness of lattice structures fabricated by SLM by using CoCrMo powder. Kim et al. [51] claimed that post-SLM sintering or jet blasting can result in localized removal of these powder off the struts without changing the macro-properties of the scaffolds.

Leary et al. [52] found that downward facing surfaces of inclined struts had higher surface roughness than upward facing surfaces. They worked on overhanging struts having inclinations of 0, 35.26, 45 and $90^{\circ}$ being made up of AlSi12Mg by using SLM. They reported that horizontal struts $\left(0^{\circ}\right.$ inclined) were not printable for any of the diameters they tested. But they reported that 
reducing the spanned distance can improve manufacturability for low inclination struts. Similar surface roughness results of Leary et al. were found by Fox et al. [53], Triantaphyllou et al. [54], Drescher et al. [55], and Suard [56]. For Ti6Al4V, cantilever strut elements were studied by Mazur et al. [57]. They varied inclination angle in the range of $0-60^{\circ}$ with $10^{\circ}$ increments, and strut diameter of $0.3-1.0 \mathrm{~mm}$ with $0.1 \mathrm{~mm}$ increments. They found out that the minimum manufacturable strut angle is $20^{\circ}$ and the minimum manufacturable strut diameter is $0.3 \mathrm{~mm}$. On the other hand, they also found out that struts as big as $3 \mathrm{~mm}$ are manufacturable while supported at both ends. But horizontal struts ended up with oversized diameters due to high powder adhesion. Weissmann et al. [58] studied vertical and $45^{\circ}$ inclined struts fabricated by SLM and Electron Beam Melting (EBM). The struts from the SLM process were closer to the target specification than the EBM-manufactured struts. The $45^{\circ}$ inclined struts had bigger deviations from the desired.

Rehme and Emmelmann [59] defined an aspect ratio as cell edge length to strut diameter to assess the manufacturability by changing strut radius, cell size, and cell type. For 316-L stainless steel, the printable range was in between 3.36 and 22.9 from relatively low to high, respectively. They also reported that printability increases when strut length gets shorter. About minimum printable radius, researchers reported several values. The successful production of strut diameter of $0.05 \mathrm{~mm}$ were achieved by VanBael et al. [43] and Hao et al. [60] stated that struts with $0.025 \mathrm{~mm}$ radius were possible. The studies investigating manufacturability of lattice structures, to authors' knowledge, concentrated on specific parameters for small variations and only for a limited number of lattice types. Only one study [59] mentions explicit limits for one machine precision and powder type. However, after its publication, other researchers $[43,60]$ reported results beating those values. Through the advancements in technology, the printable density limits are changing. On the other hand, the printable density is dependent on the specifications of each process, and in fact, each machine and the raw material used. Therefore, there is a need for a tool estimating printable density limits of lattice structures based on given conditions. In this work, the authors try to estimate those limits only by using the most fundamental parameters affecting those limits: the machine precision and powder size for different lattice types by leaving the study of other parameters to later studies. Since these are the most basic factors in terms of printability and some other parameters can be 
modeled with these, the study can be used as the basis point for further research.

\section{Problem formulation}

To create the CAD models of the lattices, a preprocessor of a commercial CAE software [61] is used together with Python scripting language to enable the algorithms presented in this study to be embodied. The unit cells with cubic outer boundary space are considered. All struts in a lattice are assumed to have the same circular cross-sectional area. Figure 1 shows the unit cell types used schematically.

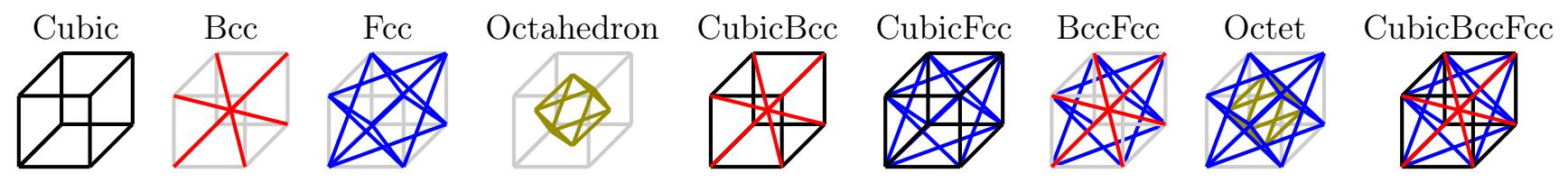

Figure 1: Unit cell types used.

The numerical prediction of the lower and higher printable density limits of lattice structures for AM problem can be stated mathematically as follows:

\begin{tabular}{lll} 
Find $:$ & $r=r_{l}^{\prime}$ & $r=r_{h}^{\prime}$ \\
& Minimizes $\rho_{\text {low }}=\rho\left(r, t, L, r_{\text {min }}\right)$ & Maximizes $\rho_{\text {high }}=\rho\left(r, t, L, V_{p}\right)$ \\
\hline
\end{tabular}

For : $\quad$ 1) $\forall t \in T \mid T=\{C u b i c, B c c, F c c$, Octahedron, CubicBcc, CubicFcc,

BccFcc, Octet, CubicBccFcc\}

2) $\forall L \mid L \in \mathbb{R}^{+}$

3) $\forall r_{\text {min }} \in \bar{r}_{\text {min }} \mid \bar{r}_{\text {min }}=\left\{r_{\text {min }} \mid r_{\text {min }} \in \mathbb{R}^{+} \& r_{\text {min }} \leq L\right\}$

4) $\forall V_{p} \in \bar{V}_{p} \mid \bar{V}_{p}=\left\{V_{p} \mid V_{p} \in \mathbb{R}^{3} \& 0<V_{p}<L^{3}\right\}$

Such That: 1) $r_{\min }=r \leq L$

1) $0<r \leq L$

2) $n_{\text {void }}=1$

3) $\pi r_{\text {ins }}^{2}$ on $\partial \Omega \geq A_{\text {min }}$

4) $\exists \Omega_{s} \mid \Omega_{s}=\left\{P \in \Omega_{v} \wedge\|P, \gamma\| \leq r_{\text {lump }}\right\}$

$\gamma: I \rightarrow \mathbb{R}^{3} \& \gamma \subset \Omega_{v} \& I=I_{v} \& \min \left(\frac{1}{\kappa(\gamma)}\right) \geq r_{l u m p}$ 
where $r$ is the strut radius; $r_{l}^{\prime}$ is the critical strut radius value related to $\rho_{\text {low }} ; \rho_{\text {low }}$ is the lowest printable relative density; $r_{h}^{\prime}$ is the critical strut radius value related to $\rho_{\text {high }} ; \rho_{\text {high }}$ is the highest printable relative density; $L$ is the cell size of a lattice; $n_{\text {void }}$ is the number of independent void volumes inside the cell; $r_{\min }$ is the minimum printable radius which is a machine specific value; $t$ is the lattice type; $V_{p}$ is the volume of the sphere circumscribing the biggest powder particle used; $r_{\text {insmax }}$ is the radius of the biggest sphere that can fit into every chunk of void volume; $A_{\text {min }}$ is the corresponding powder cross-sectional area; $\Omega_{v}$ is the void volume of a lattice; $\partial \Omega$ is the surface of the cube enclosing a lattice; $\Omega_{s}$ is a sub-volume of $\Omega_{v} ; P$ is a point; $\gamma$ is a space curve; \|\| is Euclidean distance operator; $r_{l u m p}$ is the corresponding radius of $V_{p} ; I$ is the interval over which $\gamma$ defined; $I_{v}$ is the interval over which $\Omega_{v}$ defined; $\kappa$ is the curvature of a curve at every point; $\min \left(\frac{1}{\kappa(\gamma)}\right)$ is the minimum radius of curvature of the curve $\gamma$.

More specifically, the main objective of this study is to find the minimum and the maximum printable relative density for a given lattice type, lattice size, the minimum printable radius, and the powder lump size. The strut radius values should not be smaller than zero and should not be bigger than the lattice cell size. While finding the minimum and the maximum printable relative density, the void volume should be monolithic (See the constraint 2 on the right-hand side of Eqn. 1.). One may think that a single lattice having disconnected voids can be printable as long as the voids are connected to one of the faces of the cubic envelope. However, when considering periodic lattices, and considering that the faces are symmetry planes, these disconnected voids will turn into closed cavities which entrap powder. By constraining the sub-volume formation, it is ensured that the powder can go out as long as at least one face is open. Hence, the number of void volumes inside a lattice should be equal to unity.

The powder used in the AM process has a size. Moreover, the unused powder may form lumps during printing, which in turn makes their effective radius even bigger, e.g. by partial sintering of loose particles, or by spatter from the process [62], landing on the unused powder zones. Thus, having a monolithic void volume is not enough on its own in order to take the powder out. In Figure 2, a 2D schematic shows how a powder particle can go through the void between two struts. In order to ensure that the powder in the void spaces can be evacuated after printing, the void 
volume has to have a minimum hole area which is bigger than the corresponding cross-sectional area of the powder lumps on faces of the lattices (See the constraint 3 on the right-hand side of Eqn. 1.).

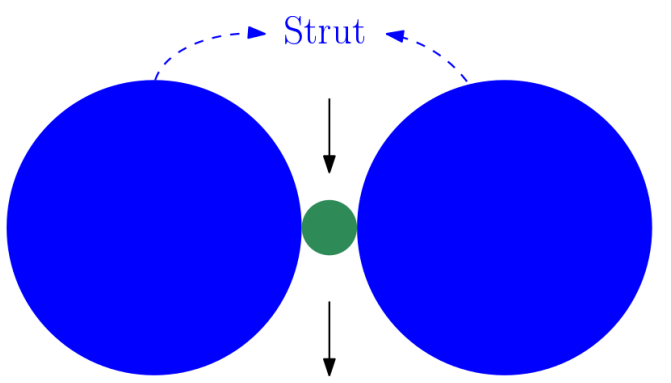

Figure 2: A 2D schematic for powder passage through the void between two struts.

Besides, there has to be enough space for powder inside the void volume to let the powder go through. To ensure so, a void sub-volume is required, whose branches' cross-sectional area has to be equal to the cross-sectional area of the powder lump, at least. A space curve, $\gamma$, is employed to define the sub-volume over the same interval of the void volume. Since the powder is a sphere, checking the cross-sectional area is not enough to prevent powder entrapment. Hence, the curvature of the space curve is also constrained as defined in the constraint 4 on the right-hand side of Eqn. 1. In other words, there should be, at least, a sub-volume, $\Omega_{s}$, inside the void volume whose interval is the same as the interval the void volume defined over so that it touches the faces of the enclosing cube. By adding the distance to the formulation of $\Omega_{s}$, a pipe-like volume whose cross-section is a circle with $r_{\text {lump }}$ is created throughout the interval. By checking the curvature of the curve, one will be sure that the powder sphere will go through the pipe-like sub-volume; therefore the void volume.

On the other hand, machines are subjected to precision constraints, as well. Hence, they have a limit of the smallest printable radius. For this reason, the smallest printable radius should be taken into account for estimating limits of being printable for a lattice. Therefore, in order to find $\rho_{\text {low }}$ for each lattice type, this constraint has to be taken into account (See the constraint-1 of Eqn. 1). 


\section{Methodology}

The problem defined in Eqn. 1 is, initially, attacked by assuming the powder as infinitesimally thin, because of the fact that the infinitesimal assumption of the powder gives the limit cases. Besides, it gives the location of the critical points which are inside the lattice at which the formation of the disconnected void volumes starts. In other words, these critical points are the separation points (bottlenecks) that keep the void volume monolithic. Then using the results of the infinitesimal problem, the problem with finite size powder is attacked.

\subsection{Infinitesimally thin powder problem}

In this scenario, it is assumed that the powder is infinitesimally thin and the machine used has infinite precision. As a result, it can be considered that any shape is printable, and the powder can be taken out from a hole as long as its area is bigger than zero regardless of however small it is. Through this problem, the maximum theoretical printable density can be investigated.

Needless to say, the lower limit, only $\rho_{l o w}(r, t, L)$, will be zero in this case. Hence, in this case, the $\rho_{\text {high }}(r, t, L)$ will be calculated. All in all, the problem reduces to the form below:

$$
\begin{array}{ll}
\text { Maximize } & \rho_{\text {high }}=\rho\left(r, t, L, A_{\min }=\pi \varepsilon^{2}\right) \\
\text { Subjected to }: & A^{f} \text { on } \partial \Omega \geq \pi \varepsilon^{2} \\
& n_{\text {void }}=1
\end{array}
$$

Since the powder is infinitesimal, there is no need to check hole area in the void volume as long as the void volume is monolithic and the void volume can reach the faces. The solution approach to this problem is to find the maximum $\rho_{\text {high }}$ using only one constraint at a time. Then, it is to choose the smaller $\rho_{\text {high }}$ among the two solutions found.

$A^{f}$ is a continuous function that shows the hole area on a face of lattice, which is a function of relative density, $\rho$, that is, in turn, a function of strut radius, $r$. To find $\rho\left(r_{h}^{\prime}\right)$, one needs to find $A^{f}\left(r_{h}^{\prime}\right)=\pi \varepsilon^{2}$. By changing the problem to $A^{f}(r)-\pi \varepsilon^{2}=0$, one can solve it by using the bisection method, which does not need to have a derivative of the function. $\varepsilon$ is an infinitesimal positive quantity, which is taken as $10^{-5}$ by considering that the precision of the software to define a geometric entity is $10^{-6}$. 
$n_{\text {void }}$ is a piece-wise function that shows the number of independent void volumes inside of the lattice, which is a function of $\rho$, which is, in turn, a function of strut radius, $r$. Then, one needs to find maximum $r$ that satisfies $n_{\text {void }}\left(r=r_{h}^{\prime}\right)=1$. A typical change of $n_{\text {void }}$ along relative radius, $\bar{r}=\frac{r}{L}$, is shown in Figure 3 .

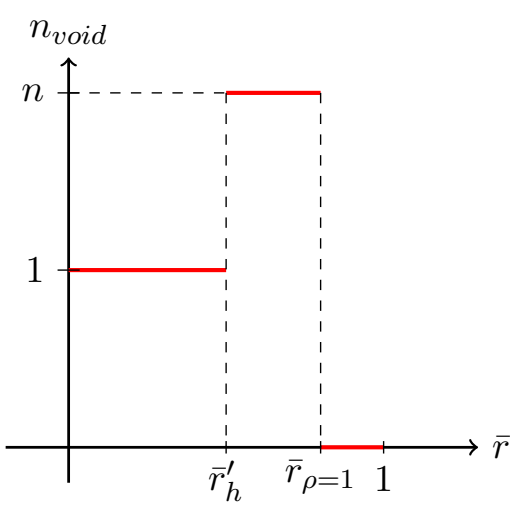

Figure 3: Typical number of void volumes versus the relative strut radius in an unit cell.

While having a function like above, $n_{\text {void }}$, at hand, finding $\bar{r}_{h}^{\prime}$ is tricky. First, the function is piecewise discontinuous. Second, it is not closed-form function of the free variable, $r$ or $\bar{r}$. At this juncture, a zeroth order method looms as the most convenient alternative, which does not require calculation of the derivatives. Therefore, the bisection method is chosen for the search of $\bar{r}_{h}^{\prime}$ for the case of $n_{\text {void }}=1$ as well. However, the algorithm needs a modification as described in the next section.

\subsubsection{Modified bisection method}

The bisection method is originally intended to find roots for continuous functions like $f(x)=0$ on the interval $\left[x_{l}, x_{u}\right]$. Nonetheless, the problem at hand is to find the discontinuity. Turning $n_{\text {void }}$ as $n_{\text {void }}:=n_{\text {void }}-1$ will not help finding $\bar{r}_{h}^{\prime}$ because of the fact that any values between 0 and $\bar{r}_{h}^{\prime}$ will be the solution of the bisection method. Therefore, the method is slightly modified to narrow the search space systematically. In Figure 4, the flow chart of its modified form is illustrated.

In Figure $4 a$ and $b$ designates the lower and upper bound of the updated interval; $c$ designates the midpoint of the interval. The modified bisection method first finds the midpoint of the interval and then evaluates it. If $f(c)=1$, then it updates the lower bound of the interval by setting the midpoint as $a=c$; if not, it updates the upper bound of the interval by setting the midpoint as 


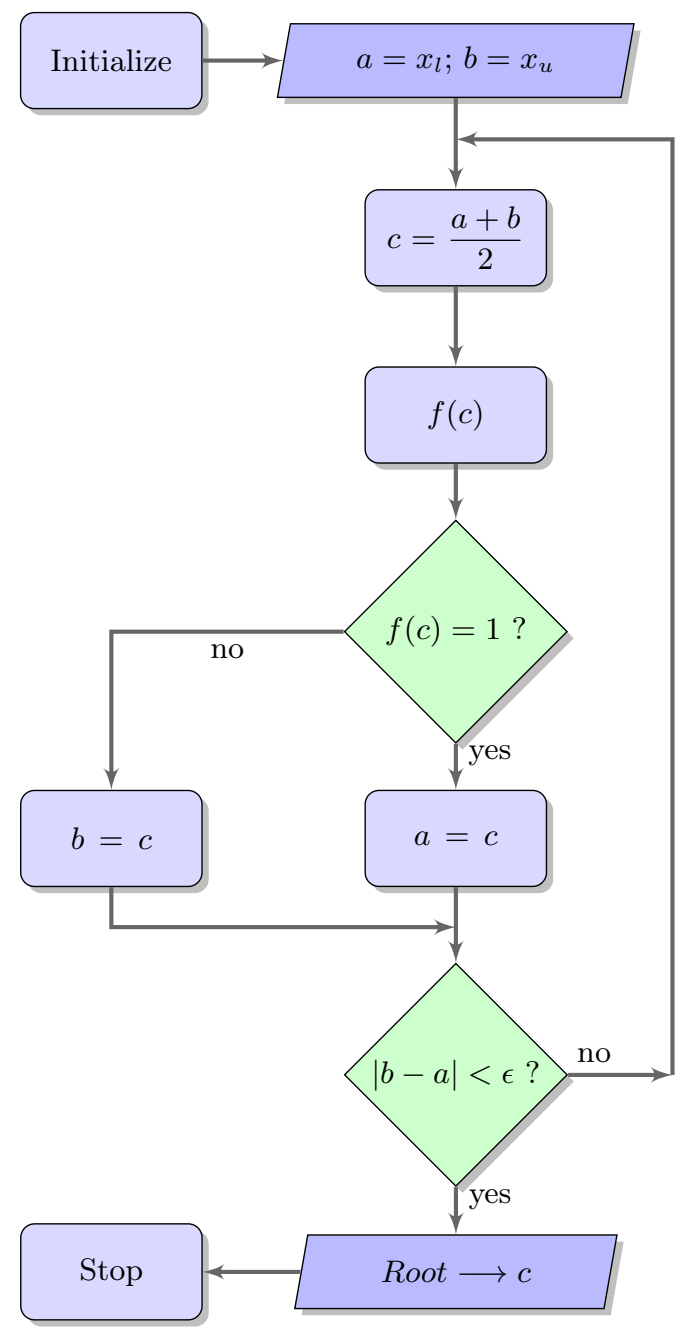

Figure 4: Flow chart of modified bisection method for function at hand which is depicted in Figure 3.

$b=c$. This loop continues until the difference between $\mathrm{b}$ and $\mathrm{a}$ is smaller than a specified positive small value, $\varepsilon$. As can be seen from Figure 4, the original algorithm is modified by changing the interval update from multiplication of function values, $f(a) f(c)<0$, to $f(c)=1$.

\subsection{Finite size powder problem}

\subsubsection{Calculation of $\rho_{\text {low }}$}

The calculation of $\rho_{\text {low }}$ is independent from $\rho_{\text {high }}$. Therefore, the problem is reduced to

$$
\begin{array}{ll}
\text { Minimize } & \rho\left(r, t, L, r_{\text {min }}\right) \\
\text { Subjected to }: & r=r_{\text {min }}
\end{array}
$$

To find $\rho_{\text {low }}$, it is only needed to calculate the relative density, $\rho$, based on the specified the 
minimum printable radius, $r_{m i n}$.

To make the calculations easier, the relative density, $\rho$, is calculated based on the relative radius, $\bar{r}$. Then a polynomial is fit to the data. By plugging the dimensionless minimum printable radius, $\frac{r_{\min }}{L}$, to the polynomial fitted, $g$, one can find the lower relative density limit, $\rho_{l o w}$.

$$
\rho_{\text {low }}=g\left(\bar{r}=\frac{r_{\min }}{L}, t, L\right)
$$

\subsubsection{Calculation of $\rho_{\text {high }}$}

Since the smallest printable radius is introduced, the lower limit of the relative density will be higher. Likewise, since the constraint of the smallest area in the void volume is imposed for taking the powder out, the higher limit of the relative density will be lower. To sum up, in this case, the printable interval of the relative density of lattice structures will be narrower than the infinitesimal powder problem.

As can be viewed from the results of the infinitesimally thin powder problem (see Table 3 in Section 4.1), there are bottlenecks in the void volume. To calculate the higher limit, $\rho_{\text {high }}$, one has to find the locations and the orientations of these bottlenecks, which are the smallest interconnecting sections that keep the void volume monolithic and determine whether the cross section lets the powder go through. From the infinitesimally thin powder problem, one already knows the positions of the bottlenecks. On both sides of these separation points (bottlenecks), the cross section increases. Therefore, checking the void volume's cross-sectional area at these points and normal to the bottleneck axis will be enough to check the powder entrapment. The simplified problem can then be stated as below:

$$
\begin{array}{ll}
\text { Maximize } & \rho\left(r, t, L, V_{p}\right) \\
\text { Subjected to }: & n_{\text {void }}=1 \\
& \pi r_{\text {ins }}^{2} \text { on } \partial \Omega \geq A_{\min } \\
& \pi r_{\text {ins }}^{2} \text { on } \text { C.P. } \geq A_{\text {min }}
\end{array}
$$

where C.P. stands for the cross plane perpendicular through the bottleneck points.

The removal of the powder is based on the fact that the monolithic void volume in each cell 
leads to an interconnected monolithic void in the multi-cell structure. Therefore the removal of the powder in the voids is possible with help of gravity, vibration and air pressure, as long as the powder particles can pass through the bottlenecks in the interconnected cell voids.
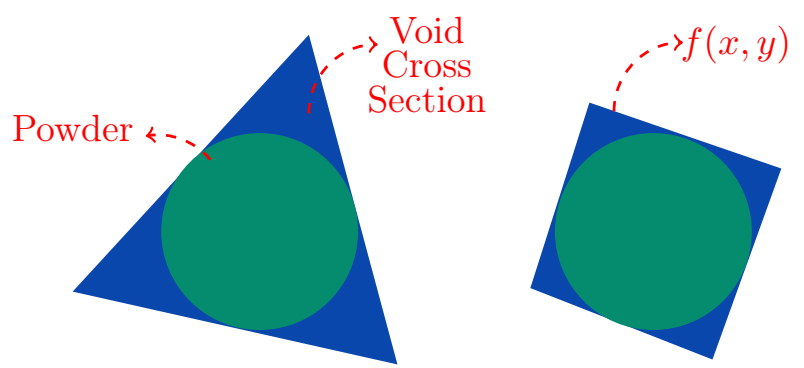

Figure 5: A few hypothetical scenarios for powder and hole cross-sectional area, which powder can be taken out.

In the infinitesimally thin powder case, the void volume is maintained together only with point contact. Slightly bigger values than the critical radius, $r_{h}^{\prime}$, will result in multiple void volumes and create twin points on each side of the newly separated void volumes. Using the CAD model of a lattice for $r_{h}^{\prime}\left(V_{p}=\frac{4}{3} \pi \varepsilon^{3}\right)$, one may pinpoint the bottlenecks and their orientations. One may partition the void volume at the desired location and at the desired orientation such that one can find the smallest cross-section using CAD representation for any given strut radius, $r$ (see Figure 3). Finding the biggest radius of a circle that can be inscribed on the smallest section of the void volume, $r_{i n s_{\max }}$, is yet another optimization problem which can be stated as follows:

$$
\begin{array}{ll}
\text { Maximize } & r_{\text {ins }} \\
\text { Such that : } & Y=\left\{(x, y) \mid x, y \in \mathbb{R} \wedge x^{2}+y^{2} \leq f(x, y)\right\} \\
& X=\left\{(x, y) \mid x, y \in \mathbb{R} \wedge\left(x-x_{G}\right)^{2}+\left(y-y_{G}\right)^{2} \leq r_{\text {ins }}^{2} \wedge(x, y) \in Y\right\}
\end{array}
$$

where $r_{i n s}$ is the radius of an inscribed circle on a arbitrary cross section through the void volume of a lattice, $\Omega ; \mathbb{R}$ is real numbers; $f(x, y)$ is the defining function of boundary of the critical cross-sectional hole shape of a lattice; $\left(x_{G}, y_{G}\right)$ is the central location of the inscribed circle.

As depicted in Figure 6, the cross-sectional shape of the void around the separation point (S.P.) changes like nested polygons depending on the strut radius, $r$, since the struts of a lattice are oriented always the same and they have the same radius. Hence, to find the $r_{i n s_{\max }}$ reduces to 


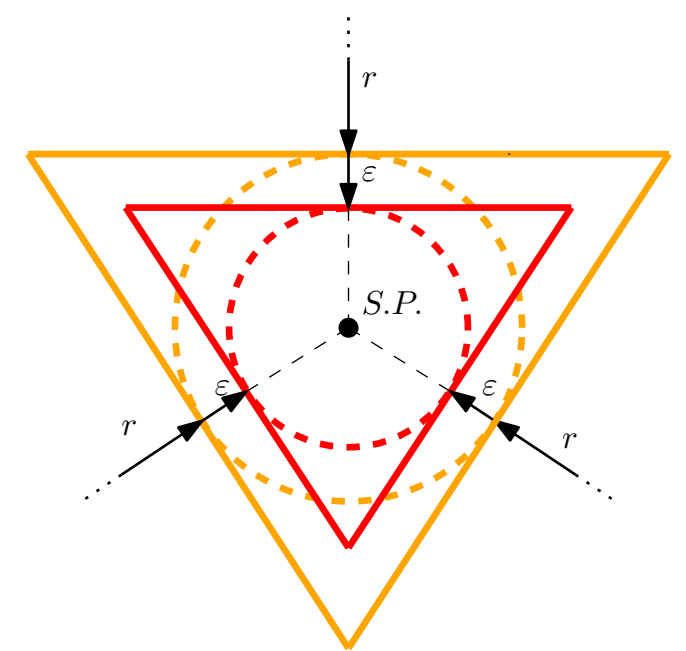

Figure 6: Typical void cross-sectional shape change in terms of strut radius around separation point.

lower $r_{h}^{\prime}\left(V_{p}=\frac{4}{3} \pi \varepsilon^{3}\right)$ in $\S 3.1$ as much as the minimum allowed circular hole area specified by $r_{\text {lump }}$. Mathematically, it can be stated as the following equation:

$$
r_{h}^{\prime}\left(V_{p}=\frac{4}{3} \pi r_{\text {lump }}^{3}\right)=r_{h}^{\prime}\left(V_{p}=\frac{4}{3} \pi \varepsilon^{3}\right)-r_{\text {lump }}
$$

In dimensionless form, $\rho_{\text {high }}$ can be found using the following equation:

$$
\rho_{\text {high }}=g\left(\frac{r_{h}^{\prime}\left(V_{p}=\frac{4}{3} \pi \varepsilon^{3}\right)}{L}-\frac{r_{l u m p}}{L}\right)
$$

where $g$ is the polynomial function fitted to $\rho=\rho(\bar{r})$.

\section{Results}

\subsection{Infinitesimally thin powder problem}

After calculating the corresponding $r_{h}^{\prime}$ values for each constraint separately (See Eqn. 2), the smaller one is taken among the two and by using that value the corresponding relative density is calculated as explained before. The resultant printable upper-density limits are depicted in Figure 7 for the lattice types given. As can be seen from the figure, $\rho_{\text {high }}$ does not depend on the lattice size, $L$. 


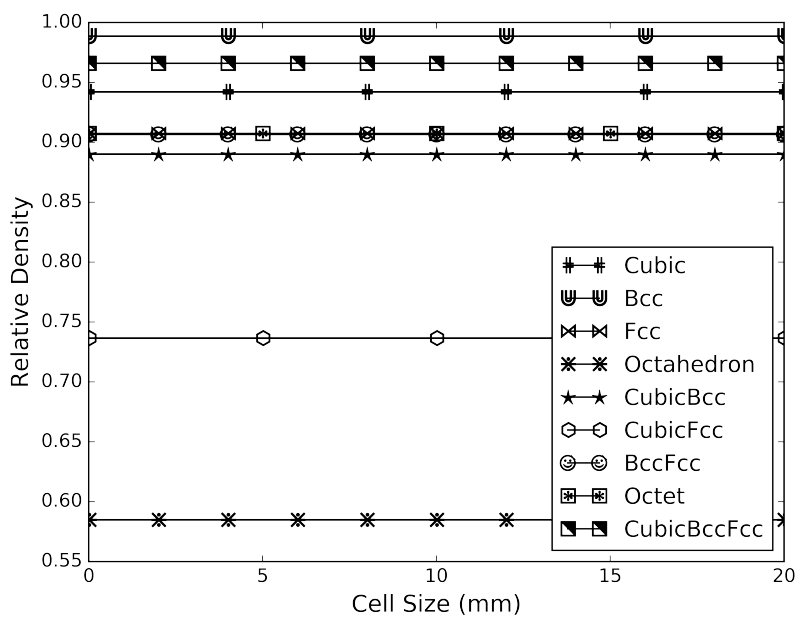

Figure 7: Upper limits for several lattice types based on the two criteria together.

In Table 1, upper density limits, $\rho_{h i g h}$, and the corresponding critical relative radius values, $r_{h}^{\prime}$, are given. The Octahedron has a very low upper limit of printable density in comparison to the others. The reason of getting low relative printable density limit for Octahedron is that Octahedron has struts surrounding an inner cavity. When the struts radius increases, the central cavity easily gets disconnected from outer cavities, which are still quite large, resulting in a low relative density limit. On the other hand, Bcc has the highest limit. Here, for Bcc lattice, the struts stem from the center and for a growing strut radius, the cavities remain connected almost up to the disappearance of the cavities.

Table 1: Critical relative radius, $\frac{r_{h}^{\prime}}{L}$, values corresponding to upper relative density limits, $\rho_{\text {high }}$.

\begin{tabular}{lcc}
\hline Lattice Type & $\frac{r_{h}^{\prime}}{L}$ & $\rho_{\text {high }}$ \\
\hline Cubic & 0.5 & 0.9420 \\
Bcc & 0.3553 & 0.9886 \\
Fcc & 0.2887 & 0.9073 \\
Octahedron & 0.2024 & 0.5849 \\
Cubic Bcc & 0.2588 & 0.8902 \\
Cubic Fcc & 0.2071 & 0.7365 \\
Bcc Fcc & 0.2142 & 0.9065 \\
Octet & 0.2041 & 0.9073 \\
Cubic Bcc Fcc & 0.2071 & 0.9660 \\
\hline
\end{tabular}

In Table 3, the corresponding solid and void volumes are graphically shown from the different 
point of views for all lattice types. As can be seen from Table 3, for Cubic, CubicFcc, and CubicBccFcc, the bottlenecks are on the face of the corresponding lattice. However, for the rest, the bottleneck is inside of the corresponding lattice.

Since $\rho=\rho(\bar{r})$ relationship will be used in all limit calculations (Not only for infinitesimal powder assumption but also for finite powder assumption as explained in $\S 3.2 .2), \rho$ versus $\bar{r}$ is plotted in Figure 8 for all lattice types. The corresponding data of the printable portion, under infinitesimal powder assumption, of $\rho=\rho(\bar{r})$ (see the right plot of Figure 8) is fitted to cubic polynomials in order to give a practical tool for designers (see Table 2). In other words, the polynomials are valid until the critical relative radius values specified for every lattice type in Table 1 . The polynomial fit is confined to the printable zone due to the fact that achieving the intended density is not possible outside of it.
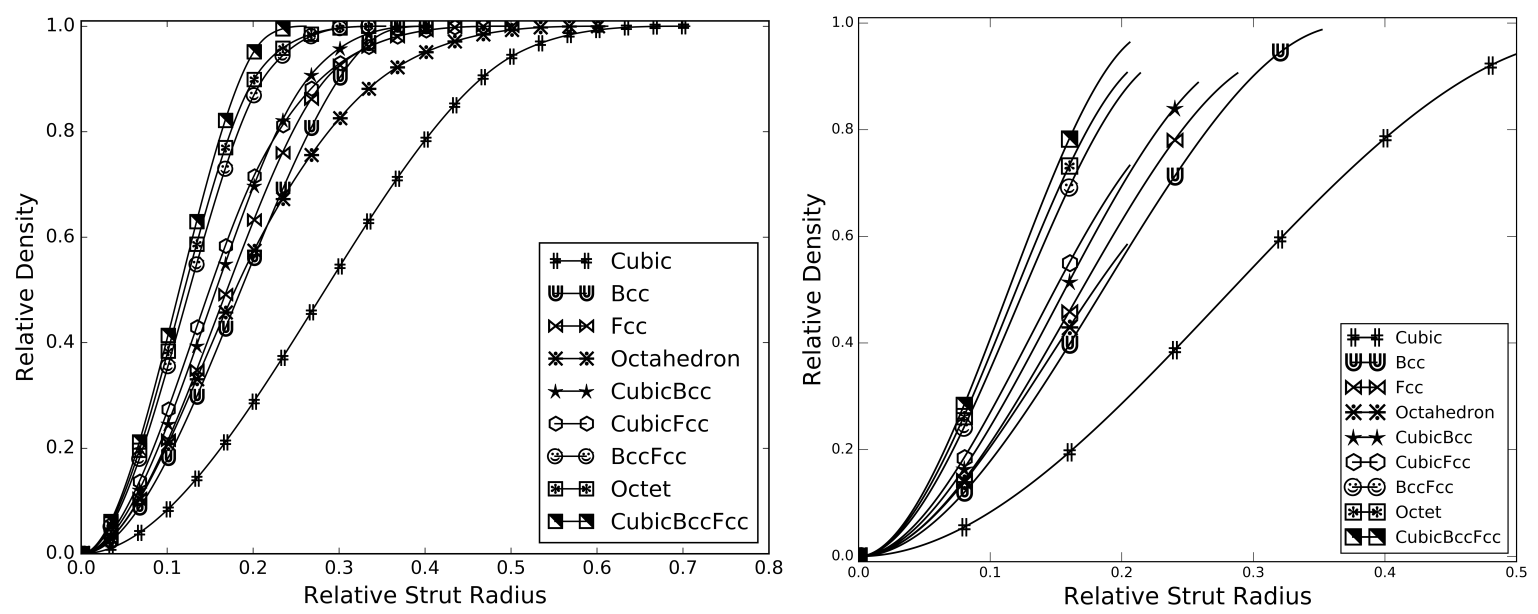

Figure 8: Relative density, $\rho$, versus relative radius, $\bar{r}$, of the struts for some lattice types: Left: Full range; Right: The printable range for the infinitesimally thin powder. 
Table 2: Coefficients of the polynomials, $g$, fitted to $\bar{r}$ for $\rho$.

\begin{tabular}{lrrr}
\hline Lattice Type & $\bar{r}^{3}$ & $\bar{r}^{2}$ & $R^{2}$ \\
\hline Cubic & -11.3138 & 9.4248 & 0.999 \\
Bcc & -39.1919 & 21.7656 & 0.999 \\
Fcc & -54.6277 & 26.6574 & 0.999 \\
Octahedron & -61.8194 & 26.6573 & 0.999 \\
CubicBcc & -69.1653 & 31.1904 & 0.999 \\
CubicFcc & -91.3146 & 36.0823 & 0.999 \\
BccFcc & -133.8197 & 48.4230 & 0.999 \\
Octet & -154.5098 & 53.3147 & 0.999 \\
CubicBccFcc & -170.5703 & 57.8478 & 0.999 \\
\hline
\end{tabular}

where $R^{2}$ is the coefficient of determination.

\subsection{Finite size powder problem}

In Figure 9, both lower and upper limits of density are plotted by assuming $r_{\min }=0.2 \mathrm{~mm}$ and $r_{l u m p}=0.2 \mathrm{~mm}$. These limits are sensitive to the cell size. As the cell size increases, the limits approach to the infinitesimal case. When it gets smaller, the two curves approach to each other, which means the printable density range gets smaller, until the two curves cross each other, which means only one density value is printable. Beyond that crossing point, no lattice having smaller density value is printable.

Figure 9 shows the curves for only $r_{\text {min }}=0.2 \mathrm{~mm}$ and $r_{\text {lump }}=0.2 \mathrm{~mm}$. However, the possible combinations of $r_{\text {min }}$ and $r_{l u m p}$ values are endless. Hence, it is necessary to build a dimensionless graph such that one can find the corresponding printable range for given $r_{\text {min }}$ and $r_{\text {lump. }}$ In Figure 10, such a graph is given, whose $\frac{r_{\min }}{L}$ and $\frac{r_{l u m p}}{L}$ are plotted on the same axis. However, completely independent values can be selected for them. The relative density values on the graph are limited up until the corresponding the highest relative density, $\rho_{\text {high }}$, of each lattice. In order to clarify how to use the figure, the printable range is found using $L=5 \mathrm{~mm}, r_{\min }=0.2 \mathrm{~mm}$, and $r_{l u m p}=0.1 \mathrm{~mm}$ for Octet lattice. The resultant range is $[0.075,0.843]$. The corresponding values are marked in Figure 10.

Based on the crossing point of the upper and lower limits, there is a relationship like below

$$
\frac{r_{\text {min }}}{L}=\frac{r_{h}^{\prime}\left(V_{p}=\frac{4}{3} \pi \varepsilon^{3}\right)}{L}-\frac{r_{l u m p}}{L}
$$


Table 3: Solid and void volumes of upper thresholds using infinitesimal powder assumption.

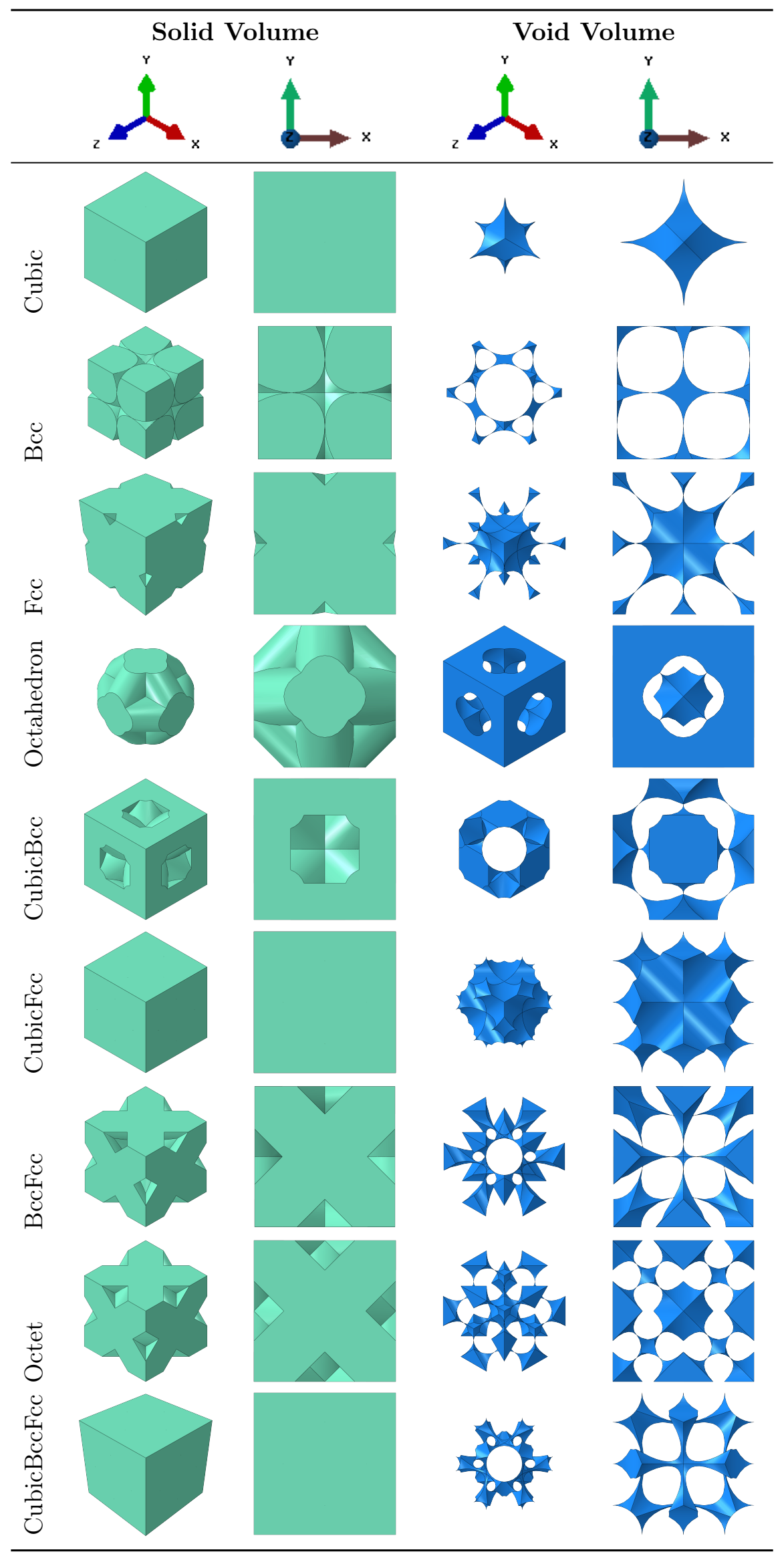




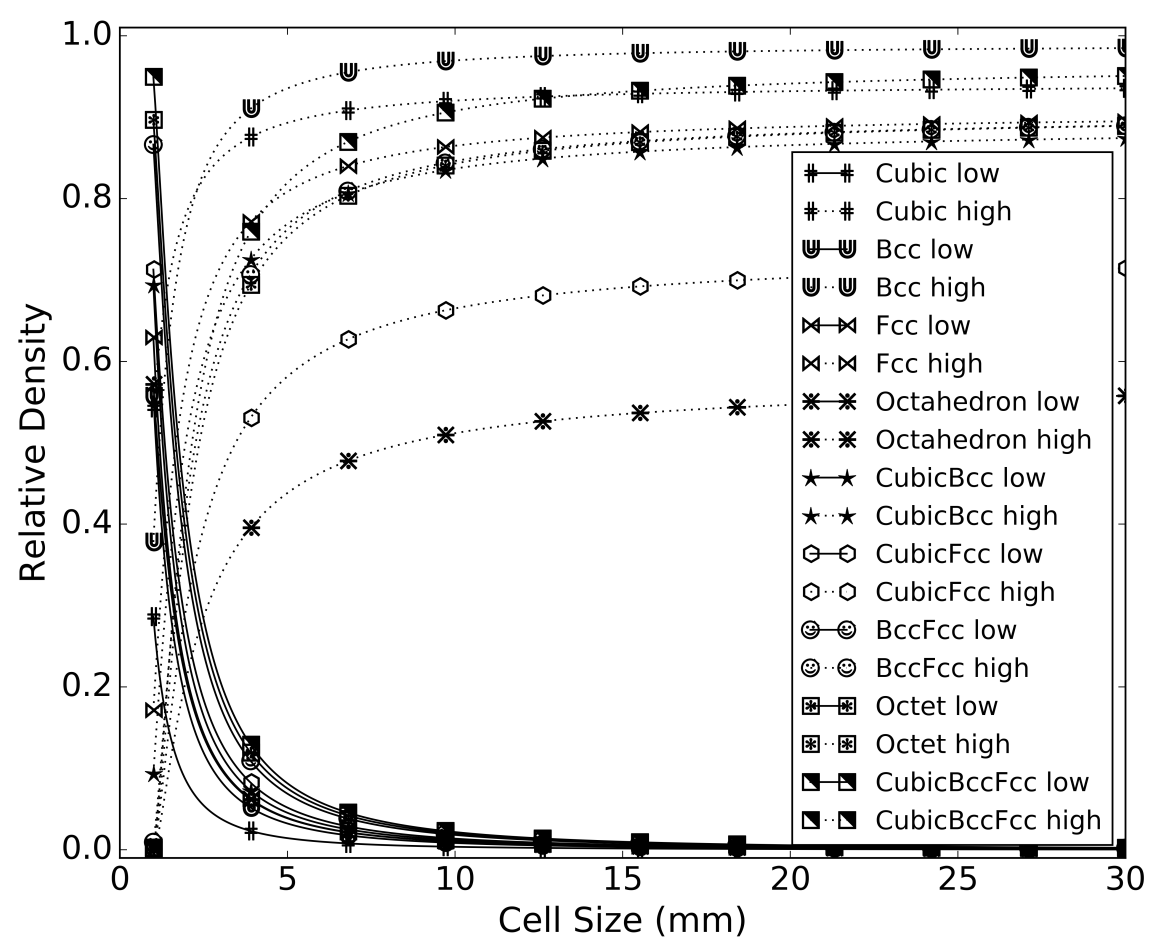

Figure 9: Thresholds of lattice structures using $r_{\min }=0.2 \mathrm{~mm}$ and $r_{l u m p}=0.2 \mathrm{~mm}$.

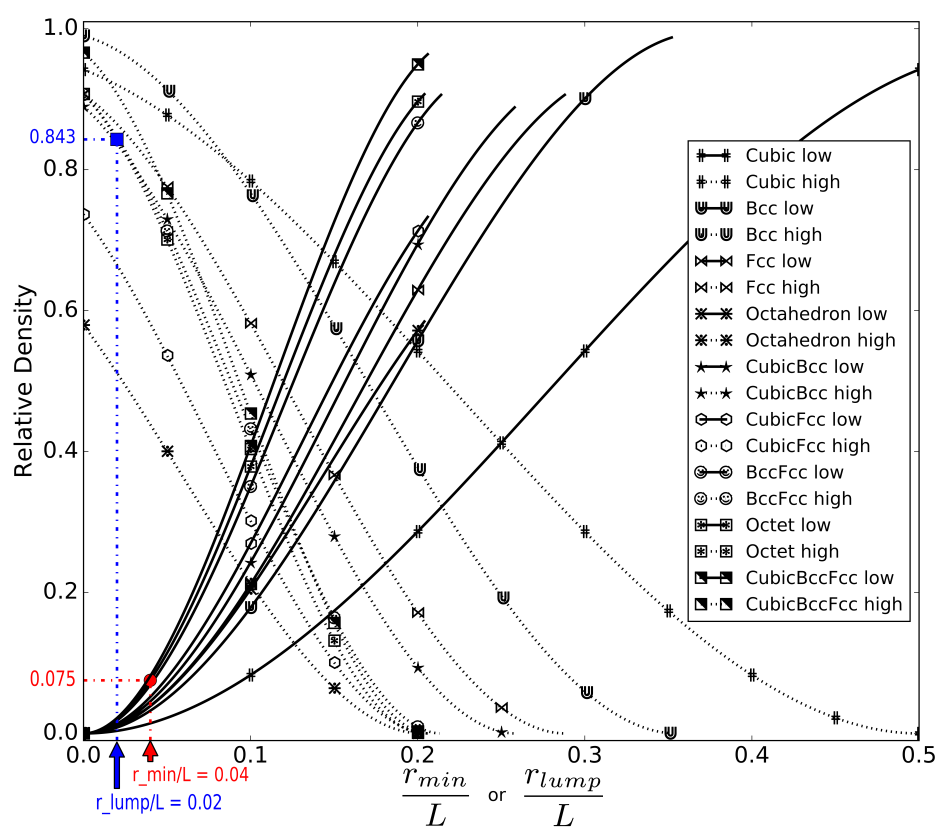

Figure 10: Printable thresholds of lattice structures in dimensionless form.

Using this equation, one can calculate the other $r$ value if one is given. Figure 11 shows the boundary of printable zone in terms of dimensionless parameters $\frac{r_{\min }}{L}$ and $\frac{r_{l u m p}}{L}$. The regions 
under the lines are the printable zones in Figure 11. If a combination is on the boundary then that means only one value can be printable.

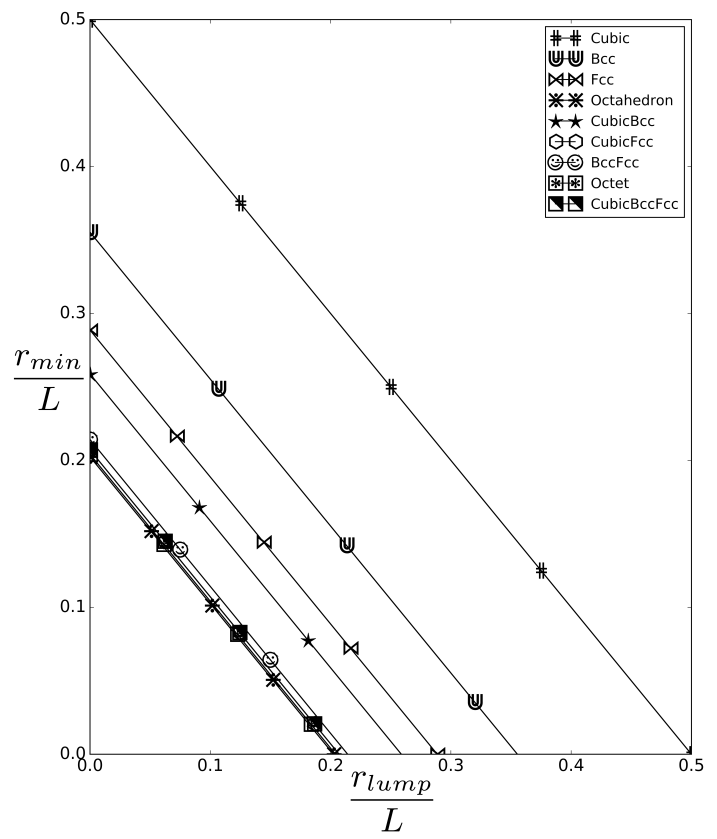

Figure 11: Boundary of printable zones in terms of $\frac{r_{\min }}{L}$ and $\frac{r_{l u m p}}{L}$.

The minimum printable feature radius based on strut inclination can be found either in the literature or by experiment. In literature, there are several minimum printable radius values in terms of strut inclination. This constraint can be treated via the machine precision (the minimum printable radius) terms in this study. That value can be plugged into the equation (Eqn. 4). Moreover, the manufacturability of horizontal struts was discussed in the literature. Mazur et al. [57] reported that by using SLM, they achieved to print horizontal struts up to $3 \mathrm{~mm}$ if they are supported at both ends. In the current state-of-the-art, the lattices having horizontal struts (e.g. Cubic, CubicBcc, CubicFcc, CubicBccFcc) are not printable unless they are smaller than 3mm for Ti6Al4V. Hence, the reader must be aware of this limitation (can be different for every powder material) when they are using Figure 10.

Powder adhesion problem can also be taken into the account by the powder lump size. As an example powder lump size can be taken as a couple of times bigger than the biggest powder size used. The exact amount has to be investigated for every material. Zhang et al. [39] suggested using 
three times bigger pore sizes. Furthermore, as reported by several authors [52-58], the difference between the realized and planned diameter of struts is a function of strut inclination. Therefore, one can expect a lattice having different diameter struts after printing even if a lattice having equal diameter struts are intended. To guess the printable limits at such conditions, one needs to estimate radius difference based on inclination angle. After taking the biggest difference expected, one can hypothetically assume a bigger powder lump as much as the biggest radius difference foreseen to find the highest printable limit.

Despite having useful estimations of the printable density range based on the assumptions made here, there is a need for a further study which includes other parameters for manufacturability of lattice structures. One can say that the real printable density range may be smaller than the one found here as explained in Eqn. 10

$$
\begin{gathered}
\rho_{\text {low }}^{\text {real }}=\rho_{\text {low }}+\rho_{\text {low }}^{\text {corr }}=\rho_{\text {low }}\left(\bar{r}+\bar{r}_{\text {corr }}\right) \\
\rho_{\text {high }}^{\text {real }}=\rho_{\text {high }}-\rho_{\text {high }}^{\text {corr }}=\rho_{\text {high }}\left(\bar{r}-\bar{r}_{\text {corr }}\right)
\end{gathered}
$$

where $\rho_{\text {low }}^{\text {real }}, \rho_{\text {high }}^{\text {real }}$ are the real lower and upper printable limits, respectively; $\rho^{\text {corr }}$ are the corrections terms.

In order to fabricate lattice structures with precise dimensions, it is important to select appropriate processing conditions. Therefore, finding optimized parameters will approach the printable limits to the ones in this study.

\section{Conclusion}

The main and novel conclusion of this study is that even if ideal conditions are assumed, there is a maximum printable density limit for each lattice type. Among the lattice types studied, Octahedron lags far behind the other lattice types on the highest printable limit while Bcc has the widest range. In the Octahedron lattice, the void enclosed by the central void quickly get separated from the relatively large remaining voids in the cell corners.

The printable range is highly dependent on the cell size as well. When unit cell size increases, the printable range widens and approaches to the limit case of infinitesimal powder case. On the other hand, the printable range gets narrower when the powder lump size increase and the machine 
precision exacerbates (i.e. increasing $r_{m i n}$ ). Dimensionless plots are created to enable finding the printable range for a given cell type, cell size, the minimum printable radius and lump size.

This approach for finding the printable density limits is valid for the lattices having a circular cross-section, and the same radius for all their struts. For the lattices having cross-sections other than circle and having the same dimension for all their struts, this approach can also be used by assuming the strut radius, $r$, to be equal to the radius of the circumscribing circle of the crosssection. But, in that case, this approach can be a conservative estimation of the relative density limits. Yet, it needs verification. For better approximation, the problem defined in Eqn. 6 has to be solved.

Although printable limits based on only two parameters have been found in this work, further studies are needed on other process parameters for exact limits. It is easily foreseeable that the real printable density range will be narrower than the ones found in this work. Furthermore, finding the optimized process parameters or the advancement of technology will approach the printable density limits to the ones found in this paper. Therefore, this study can be taken as a basis point for further investigations.

\section{Acknowledgement}

The work leading to this publication has been funded by "M3AMCAE" project, which fits in the MacroModelMat (M3) research program funded by SIM (Strategic Initiative Materials in Flanders) and VLAIO (Flanders Innovation \& Entrepreneurship).

Siemens PLM Softwares and Materialise are gratefully acknowledged for being partners in this project.

The second author acknowledges the sabbatical research fellowship awarded by the Mexican Research Council (CONACYT) and the invitation by UGent and its partners to collaborate in the M3AMCAE project.

\section{References}

[1] T. Bitzer. Honeycom technology. Cahpmann \& Hall, 1997. 
[2] David J. Sypeck and Haydn N. G. Wadley. Cellular metal truss core sandwich structures. Advanced Engineering Materials, 4(10), 2002.

[3] F. Cote, V.S. Deshpande, N.A. Fleck, and A.G. Evans. The compressive and shear responses of corrugated and diamond lattice materials. International Journal of Solids and Structures, 43(20):6220 - 6242, 2006.

[4] Gregory W. Kooistra and Haydn N.G. Wadley. Lattice truss structures from expanded metal sheet. Materials E Design, 28(2):507 - 514, 2007.

[5] J. Cochran, K.J. Lee, D. McDowell, T Sanders, B. Church, J. Clark, B. Dempsey, A. Hayes, K. Hurysz, T. McCoy, J. Nadler, R. Oh, W. Seay, and B. Shapiro. Low density monolithic metal honeycombs by thermal chemical processing. In Fourth Conference on Aerospace Materials, Processes and Environmental Technology, Huntsville, Alabama, 2000.

[6] D. J. Sypeck and H. N. G. Wadley. Multifunctional microtruss laminates: Textile synthesis and properties. Journal of Materials Research, 16:890-897, 32001.

[7] Douglas T Queheillalt, Vvikram S. Deshpande, and Haydnn. G. Wadley. Truss waviness effects in cellular lattice structures. Journal of Mechanics of Materials and Structures, 2(9):1657-1675, 2007.

[8] V. S. Deshpande and N. A. Fleck. Collapse of truss core sandwich beams in 3-point bending. International Journal of Solids and Structures, 38(36-37):6275-6305, 2001.

[9] J.C. Wallach and L.J. Gibson. Mechanical behavior of a three-dimensional truss material. International Journal of Solids and Structures, 38(40-41):7181 - 7196, 2001.

[10] S. Chiras, D.R. Mumm, A.G. Evans, N. Wicks, J.W. Hutchinson, K. Dharmasena, H.N.G. Wadley, and S. Fichter. The structural performance of near-optimized truss core panels. International Journal of Solids and Structures, 39(15):4093 - 4115, 2002.

[11] J. Wang, A. G. Evans, K. Dharmasena, and H. N. G. Wadley. On the performance of truss panels with kagome cores. International Journal of Solids and Structures, 40(25):6981-6988, 2003.

[12] W. X. Wang, D. M. Luo, Y. Takao, and K. Kakimoto. New solution method for homogenization analysis and its application to the prediction of macroscopic elastic constants of materials with periodic microstructures. Computers \& Structures, 84(15-16):991-1001, 2006.

[13] A. Askar and A. S. Cakmak. A structural model of a micropolar continuum. International Journal of Engineering Science, 6(10):583-\&, 1968.

[14] Z.P. Bazant and M. Christensen. Analogy between micropolar continuum and grid frameworks under initial stress. International Journal of Solids and Structures, 8(3):327 - 346, 1972.

[15] J. M. Guedes and N. Kikuchi. Preprocessing and postprocessing for materials based on the homogenization method with adaptive finite-element methods. Computer Methods in Applied Mechanics and Engineering, 83(2):143-198, 1990.

[16] I. G. Masters and K. E. Evans. Models for the elastic deformation of honeycombs. Composite Structures, 35(4):403-422, 1996. 
[17] L. Gibson and M. Ashby. Cellular Solids:Structure and Properties. Cambridge University Press, 1997.

[18] B. Hassani and E. Hinton. A review of homogenization and topology optimization i - homogenization theory for media with periodic structure. Computers $\&$ Structures, 69(6):707-717, 1998.

[19] B. Hassani and E. Hinton. A review of homogenization and topology optimization ii - analytical and numerical solution of homogenization equations. Computers \& Structures, 69(6):719-738, 1998.

[20] J.Y. Chen, Y. Huang, and M. Ortiz. Fracture analysis of cellular materials: A strain gradient model. Journal of the Mechanics and Physics of Solids, 46(5):789 - 828, 1998.

[21] X. L. Wang and W. J. Stronge. Micropolar theory for two-dimensional stresses in elastic honeycomb. Proceedings of the Royal Society of London A: Mathematical, Physical and Engineering Sciences, 455(1986):2091-2116, 1999.

[22] R.M. Christensen. Mechanics of cellular and other low-density materials. International Journal of Solids and Structures, 37(1-2):93 - 104, 2000.

[23] E. W. Andrews, G. Gioux, P. Onck, and L. J. Gibson. Size effects in ductile cellular solids. part ii: experimental results. International Journal of Mechanical Sciences, 43(3):701-713, 2001.

[24] V.S. Deshpande, N.A. Fleck, and M.F. Ashby. Effective properties of the octet-truss lattice material. Journal of the Mechanics and Physics of Solids, 49(8):1747 - 1769, 2001.

[25] W. E. Warren and E. Byskov. Three-fold symmetry restrictions on two-dimensional micropolar materials. European Journal of Mechanics a-Solids, 21(5):779-792, 2002. 1873-7285.

[26] A. J. Wang and D. L. McDowell. In-plane stiffness and yield strength of periodic metal honeycombs. Journal of Engineering Materials and Technology, 126(2):137-156, 2004. 10.1115/1.1646165.

[27] R. S. Kumar and D. L. McDowell. Generalized continuum modeling of 2-d periodic cellular solids. International Journal of Solids and Structures, 41(26):7399-7422, 2004.

[28] Z. Fang. Asymptotic homogenization and numerical implementation to predict the effective mechanical properties for electromagnetic composite conductor. Journal of Composite Materials, 38(16):1371-1385, aug 2004.

[29] Z. Fang, B. Starly, and W. Sun. Computer-aided characterization for effective mechanical properties of porous tissue scaffolds. Computer-Aided Design, 37(1):65-72, 2005.

[30] D. Mohr. Mechanism-based multi-surface plasticity model for ideal truss lattice materials. International Journal of Solids and Structures, 42(11-12):3235-3260, 2005.

[31] M. F. Ashby. The properties of foams and lattices. Philosophical Transactions of the Royal Society aMathematical Physical and Engineering Sciences, 364(1838):15-30, 2006.

[32] Mulalo Doyoyo and Jong Wan Hu. Plastic failure analysis of an auxetic foam or inverted strut lattice under longitudinal and shear loads. Journal of the Mechanics and Physics of Solids, 54(7):1479 - 1492, 2006.

[33] Choon Chiang Foo, Gin Boay Chai, and Leong Keey Seah. Mechanical properties of nomex material and nomex honeycomb structure. Composite Structures, 80(4):588-594, 2007.

[34] S. Demiray, W. Becker, and J. Hohe. Numerical determination of initial and subsequent yield surfaces of open-celled model foams. International Journal of Solids and Structures, 44(7-8):2093-2108, 2007. 
[35] K. Sab and F. Pradel. Homogenisation of periodic cosserat media. IJCAT, 34(1):60, 2009.

[36] Alexander L. Kalamkarov, Igor V. Andrianov, and Vladyslav V. Danishevs'kyy. Asymptotic homogenization of composite materials and structures. Applied Mechanics Reviews, 62(3), 2009.

[37] Chunze Yan, Liang Hao, Ahmed Hussein, Philippe Young, and David Raymont. Advanced lightweight 3161 stainless steel cellular lattice structures fabricated via selective laser melting. Materials $\mathcal{E}$ Design, 55:533-541, 2014. 1873-4197.

[38] T.F. Zhao, C.Q. Chen, and Z.C. Deng. Elastoplastic properties of transversely isotropic sintered metal fiber sheets. Materials Science and Engineering: A, 662:308 - 319, 2016.

[39] S. Zhang, Q. Wei, and L. Cheng. Effects of scan line spacing on pore characteristics and mechanical properties of porous ti6al4v implants fabricated by selective laser melting. Materials Design, 63:185-93, 2014.

[40] SL Sing, WY Yeong, FE Wiria, and BY Tay. Characterization of titanium lattice structures fabricated by selective laser melting using an adapted compressive test method. Exp Mechan, 56:735-748, 2015.

[41] Loong-Ee Loh, Chee-Kai Chua, Wai-Yee Yeong, Jie Song, Mahta Mapar, Swee-Leong Sing, Zhong-Hong Liu, and Dan-Qing Zhang. Numerical investigation and an effective modelling on the selective laser melting (slm) process with aluminium alloy 6061. International Journal of Heat and Mass Transfer, 80:288 - 300, 2015.

[42] Chunze Yan, Liang Hao, Ahmed Hussein, and David Raymont. Evaluations of cellular lattice structures manufactured using selective laser melting. International Journal of Machine Tools \& Manufacture, 62:32-38, 2012.

[43] S. Van Bael, G. Kerckhofs, M. Moesen, G. Pyka, J. Schrooten, and J.P. Kruth. Micro-ct-based improvement of geometrical and mechanical controllability of selective laser melted ti6al4v porous structures. Materials Science and Engineering: A, 528(24):7423 - 7431, 2011.

[44] D. K. Pattanayak, T. Matsushita, H. Takadama, A. Fukuda, M. Takemoto, S. Fujibayashi, K. Sasaki, N. Nishida, T. Nakamura, , and T. Kokubo. Fabrication of bioactive porous ti metal with structure similar to human cancellous bone by selective laser melting. Bioceramics Development and Applications, 1:1-3, 2011.

[45] C Qiu, S Yue, and NJE Adkins. Influence of processing conditions on strut structure and compressive properties of cellular lattice structures fabricated by selective laser melting. Mat Sci Eng A, 628:188-97, 2015.

[46] S. Tsopanos, R. A. W. Mines, S. McKown, Y. Shen, W. J. Cantwell, W. Brooks, and C. J. Sutcliffe. The influence of processing parameters on the mechanical properties of selectively laser melted stainless steel microlattice structures. J Manuf Sci Eng, 132(041011), 2010.

[47] Di Wang, Yongqiang Yang, Ruicheng Liu, Dongming Xiao, and Jianfeng Sun. Study on the designing rules and processability of porous structure based on selective laser melting (slm). Journal of Materials Processing Technology, 213(10):1734 - 1742, 2013.

[48] Eberhard Abele, Hanns A. Stoffregen, Klaus Klimkeit, Holger Hoche, and Matthias Oechsner. Optimisation of process parameters for lattice structures. Rapid Prototyping Journal, 21(1):117-127, 2015.

[49] E. Sallica-Leva, A.L. Jardini, and J.B. Fogagnolo. Microstructure and mechanical behavior of porous ti-6al-4v parts obtained by selective laser melting. Journal of the Mechanical Behavior of Biomedical Materials, 26:98 - 
$108,2013$.

[50] K. Hazlehurst, C.J. Wang, and M. Stanford. Evaluation of the stiffness characteristics of square pore cocrmo cellular structures manufactured using laser melting technology for potential orthopaedic applications. Materials Design, 51:949-955, 2013.

[51] Taek Bo Kim, Sheng Yue, Ziyu Zhang, Eric Jones, Julian R. Jones, and Peter D. Lee. Additive manufactured porous titanium structures: Through-process quantification of pore and strut networks. Journal of Materials Processing Technology, 214(11):2706 - 2715, 2014.

[52] Martin Leary, MaciejMazur, Joe Elambasseril, Matthew McMillan, Thomas Chirent, Yingying Suna, Ma Qian, Mark Eastona, and Milan Brandt. Selective laser melting (slm) of alsi12mg lattice structures. Materials and Design, 98:344-357, 2016.

[53] Jason C. Fox, Shawn P. Moylan, and Brandon M. Lane. Effect of process parameters on the surface roughness of overhanging structures in laser powder bed fusion additive manufacturing. Procedia CIRP, 45:131 - 134, 2016.

[54] Andrew Triantaphyllou, Claudiu L Giusca, Gavin D Macaulay, Felix Roerig, Matthias Hoebel, Richard K Leach, Ben Tomita, and Katherine A Milne. Surface texture measurement for additive manufacturing. Surface Topography: Metrology and Properties, 3(2):024002, 2015.

[55] Philipp Drescher, Thomas Reimann, and Hermann Seitz. Investigation of powder removal of net-structured titanium parts made from electron beam melting. International Journal of Rapid Manufacturing, Vol. 4(2), 2014 .

[56] M. Suard, G. Martin, P. Lhuissier, R. Dendievel, F. Vignat, J.-J. Blandin, and F. Villeneuve. Mechanical equivalent diameter of single struts for the stiffness prediction of lattice structures produced by electron beam melting. Additive Manufacturing, 8:124 - 131, 2015.

[57] Maciej Mazur, Martin Leary, Shoujin Sun, Martin Vcelka, Darpan Shidid, and Milan Brandt. Deformation and failure behaviour of ti-6al-4v lattice structures manufactured by selective laser melting (slm). Int J Adv Manuf Technol, 84:1391-1411, 2016.

[58] Volker Weißmann, Philipp Drescher, Rainer Bader, Hermann Seitz, Harald Hansmann, and Nico Laufer. Comparison of single ti6al4v struts made using selective laser melting and electron beam melting subject to part orientation. Metals, 7(3):91-113, 2017.

[59] O. Rehme and C. Emmelmann. Rapid manufacturing of lattice structures with selective laser melting. In Proceedings of SPIE 610\%: 61070K. 1-61070K. 12., 2006.

[60] L. Hao, D. Raymont, C. Yan, A. Hussein, and P. Young. Design and additive manufacturing of cellular lattice structures. Innovative Developments in Virtual and Physical Prototyping, page 249-254, 2011.

[61] Abaqus CAE User's Guide.

[62] Di Wang, Shibiao Wu, Fan Fu, Shuzhen Mai, Yongqiang Yang, Yang Liu, and Changhui Song. Mechanisms and characteristics of spatter generation in $\{\mathrm{SLM}\}$ processing and its effect on the properties. Materials $\mathcal{G}$ Design, $117: 121-130,2017$. 